\title{
Poll results: look who's doping
}

\section{In January, Nature launched an informal survey into readers' use of cognition-enhancing drugs. Brendan Maher has waded through the results and found large-scale use and a mix of attitudes towards the drugs.}

T he US National Institutes of Health is to crack down on scientists 'brain doping' with performance-enhancing drugs such as Provigil and Ritalin, a press release declared last week. The release, brainchild of evolutionary biologist Jonathan Eisen of the University of California, Davis, turned out to be an April Fools' prank. And the World Anti-Brain Doping Authority website that it linked to was likewise fake. But with a number of co-conspirators spreading rumours about receiving anti-doping affidavits with their first R01 research grants, the ruse no doubt gave pause to a few of the respondents to Nature's survey on readers' use of cognition-enhancing drugs.

The survey was triggered by a Commentary by behavioural neuroscientists Barbara Sahakian and Sharon MoreinZamir of the University of Cambridge, UK, who had surveyed their colleagues on the use of drugs that purportedly enhance focus and attention (Nature 450, 1157-1159; 2007). In the article, the two scientists asked readers whether they would consider "boosting their brain power" with drugs. Spurred by the tremendous response, Nature ran its own informal survey. 1,400 people from 60 countries responded to the online poll.

We asked specifically about three drugs: methylphenidate (Ritalin), a stimulant normally used to treat attention-deficit hyperactivity disorder but well-known on college campuses as a 'study aid'; modafinil (Provigil), prescribed to treat sleep disorders but also used off-label to combat general fatigue or overcome jet lag; and beta blockers, drugs

\section{TRENDS IN USE OF NEUROENHANCERS}

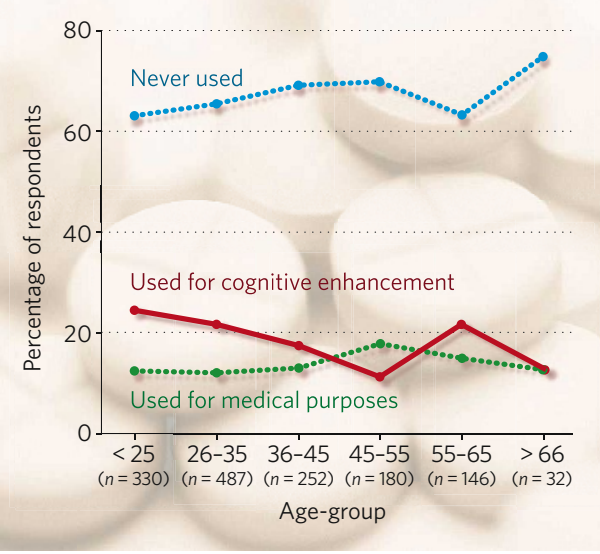

prescribed for cardiac arrhythmia that also have an anti-anxiety effect. Respondents who had not taken these drugs, or who had taken them for a diagnosed medical condition were directed straight to a simple questionnaire about general attitudes. Those who revealed that they had taken these drugs, or others, for non-medical, cognition-enhancing purposes

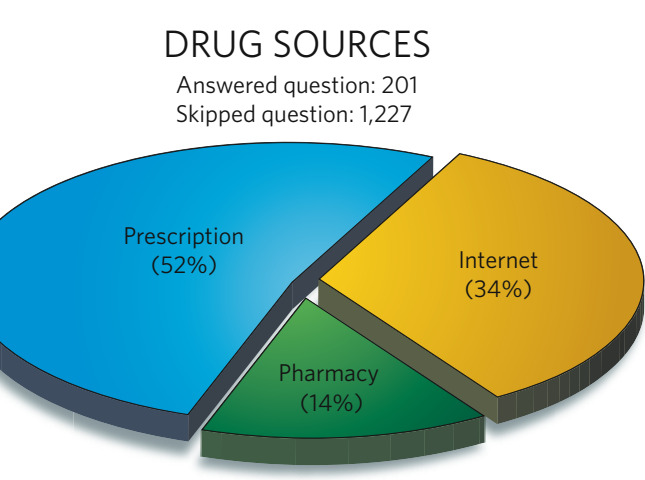

were asked several additional questions about their use. Here's what they had to say:

One in five respondents said they had used drugs for non-medical reasons to stimulate their focus, concentration or memory. Use did not differ greatly across age-groups (see line graph, left), which will surprise some. Nora Volkow, director of the National Institute on Drug Abuse (NIDA) in Bethesda, Maryland, says that household surveys suggest that stimulant use is highest in people aged 18-25 years, and in students.

For those who choose to use, methylphenidate was the most popular: $62 \%$ of users reported taking it. $44 \%$ reported taking modafinil, and $15 \%$ said they had taken beta blockers such as propanolol, revealing an overlap between drugs. 80 respondents specified other drugs that they were taking. The most common of these was adderall, an amphetamine similar to methylphenidate. But there were also reports of centrophenoxine, piractem, dexedrine and various alternative medicines such as ginkgo and omega-3 fatty acids.

The most popular reason for taking the drugs was to improve concentration. Improving focus for a specific task (admittedly difficult to distinguish from concentration) ranked a close second and counteracting jet lag ranked fourth, behind 'other' which received a few interesting reasons, such as "party", "house cleaning" and "to actually see if there was any validity to the afore-mentioned article".

Our question on frequency of use, for those who took drugs for non-medical purposes, revealed an even split between those who took them daily, weekly, monthly, or no more than once a year. Roughly half reported unpleasant side effects, and some discontinued use because of them. Some might expect that negative side effects would correlate positively with a low frequency of use, but that doesn't seem to be the case in our sample (see bar graph, below). Reported side effects included headaches, jitteriness, anxiety and sleeplessness.

Neuroscientist Anjan Chatterjee of the University of Pennsylvania in Philadelphia predicts a rise in the use of these drugs and other neuroenhancing products and procedures as they become available (A. Chatterjee Cam. Q. Healthc. Ethics 16, 129-137; 2007). Like the rise in cosmetic surgery, use of cognitive enhancers is likely to increase as bioethical and psychological concerns are overcome (see 'Worrying words') and as the products gain cultural acceptance. One difference, Chatterjee says, is that use of cognitive enhancers doesn't rely on training of medical specialists such as surgeons. Internet availability will also greatly accelerate use, he says.

Our poll found that one-third of the drugs being used for non-medical purposes were purchased over the Internet (see pie chart). The rest were obtained from pharmacies or on prescription. It is unclear whether the prescribed

\section{EFFECT OF DOSE ON SIDE EFFECTS}

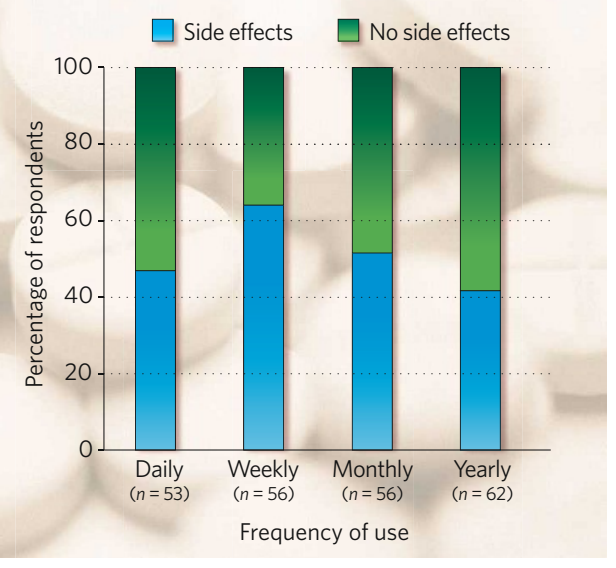


